\title{
Atrial fibrillation I: A new classification system
}

\author{
James L. Cox, MD
}

See related editorial on page 1693.
From the Division of Cardiothoracic Surgery, Washington University School of Medicine, St Louis, Mo.

Received for publication May 30, 2003; accepted for publication June 9, 2003.

Address for reprints: James L. Cox, MD, 13523 Rosewood Ln, Naples, FL 34119 (E-mail: jamescoxmd@aol.com).

J Thorac Cardiovasc Surg 2003;126: 1686-92

Copyright $\odot 2003$ by The American Association for Thoracic Surgery

$0022-5223 / 2003 \$ 30.00+0$

doi:10.1016/j.jtcvs.2003.06.002

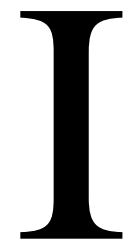

$\mathrm{n}$ the past, many relatively simple electrophysiologic concepts were made to seem complex because of the confusing and inconsistent terminology used to describe them. For example, the terms nonuniform refractoriness, inhomogeneous recovery, nonuniform repolarization, dispersion of refractoriness, and desynchronized repolarization all refer to the same electrophysiologic abnormality. We are now facing a similar problem with atrial fibrillation. Previously, atrial fibrillation could be treated within acceptable standards by physicians who possessed only a superficial understanding of the arrhythmia itself because the only options for treatment were rhythm-control or rate-control drugs. However, the recent introduction of surgical and catheter interventional treatment for atrial fibrillation has changed the requirements for therapeutic competence and, as a result, has generated the usual bewildering terminology that occurs when an entity is poorly understood and has no accurate classification system.

The confusing terminology surrounding atrial fibrillation has resulted in faulty concepts, unsound treatment schemes, and misleading claims regarding the results of interventional therapy. This problem stems primarily from the vague meaning and multiple uses of the term chronic. The descriptors paroxysmal and chronic have traditionally been used to characterize atrial fibrillation clinically, although their precise meaning has never been clarified. Most authors, including this one, have used the term paroxysmal to denote episodic or intermittent atrial fibrillation and the term chronic to denote continuous, unrelenting atrial fibrillation, both terms being applied without regard to how long the patient had the fibrillation. Recently, however, the term chronic has been used to describe atrial fibrillation of long duration, regardless of whether the atrial fibrillation is intermittent or continuous.

Because chronic in other areas of medicine means long standing, it is understandable that the term has evolved to describe long-standing atrial fibrillation. However, because chronic, when applied to atrial fibrillation, has traditionally meant continuous, its new use has led to an unfortunate and potentially dangerous confusion regarding the results of various types of interventional therapy. Moreover, our cardiology colleagues have recently proposed a new classification system for atrial fibrillation ${ }^{1}$ that includes the additional terms persistent and permanent, which, unfortunately, do not describe unique electrophysiologic characteristics and therefore only serve to cloud the issue further.

The optimal treatment of atrial fibrillation depends on a proper understanding of the electrophysiologic basis of each of its clinical manifestations. It is fortuitous that in the case of atrial fibrillation, both its clinical manifestation and its proper interventional treatment are dictated by its underlying electrophysiology. Unfortunately, accuracy in diagnosis and interventional treatment are impossible using terms such as chronic, paroxysmal, persistent, and permanent to characterize atrial fibrillation because these terms do not discriminate between the different types of atrial fibrillation, and they also mean different things to different people. The confusion in diagnosis (and therefore in the subsequent reporting of interventional results) caused by this contemporary terminology can be illustrated in the following hypothetical cases.

Suppose that a patient has had multiple, recurrent, self-terminating bouts of atrial fibrillation for the past 25 years despite medical therapy and several unsuccessful electrical cardioversion attempts. Is this chronic atrial fibrillation because it has been present for a long time? Is it paroxysmal atrial fibrillation because it is intermittent in nature? Is it persistent atrial fibrillation because it persists despite medical therapy 
and cardioversion? Is it permanent atrial fibrillation because it is long standing and cannot be ablated by medicines or cardioversion? Is the underlying electrophysiologic abnormality in such a patient related to pulmonary vein triggers, nonpulmonary vein triggers, pulmonary vein tachycardia, electrical remodeling, and/or self-sustained atrial macroreentry? Is the proper interventional treatment for this patient simple pulmonary vein isolation, the maze procedure, or something in between?

Conversely, suppose that a patient has had continuous, unrelenting atrial fibrillation for the past 7 months despite maximum medical therapy and several unsuccessful electrical cardioversion attempts. Is the diagnosis chronic atrial fibrillation because it is continuous? Is it persistent or permanent atrial fibrillation because it is resistant to medical therapy and cardioversion? Is it short-term chronic atrial fibrillation because it is both continuous and of relatively short-term duration? What is the underlying electrophysiologic abnormality in this patient and what should be the treatment goal of an interventional cardiologist or arrhythmia surgeon? Should the interventional-surgical approach be different for this patient and the first one described above?

The proper treatment of both of these patients begins with an accurate diagnosis, one that is based on the underlying electrophysiology rather than on some arbitrary and meaningless time scale relating to the duration of the arrhythmia. It is important to understand that in the absence of gross enlargement of the atria, the duration of atrial fibrillation has no effect whatsoever on the results of interventional catheter or surgical treatment.

These problems can be solved by the institution of the simple classification system that follows. This classification defines both the clinical presentation and the electrophysiologic basis of each type of atrial fibrillation and in turn dictates the proper goal of interventional therapy, surgical therapy, or both for any patient presenting with atrial fibrillation.

\section{Clinical Presentation}

Atrial fibrillation presents clinically in one of 2 ways. The patient either has atrial fibrillation all of the time or not. If the atrial fibrillation is present all of the time, it is by definition continuous. If the atrial fibrillation is not present all of the time, it is by definition intermittent. Therefore all atrial fibrillation can be classified as either intermittent atrial fibrillation or continuous atrial fibrillation.

This simple classification correctly implies that there is a fundamental pathophysiologic difference in the atrial fibrillation of a patient who presents with intermittent episodes and one who presents with continuous arrhythmia and that all other differences in their atrial fibrillation are irrelevant in terms of how they should be treated interventionally.

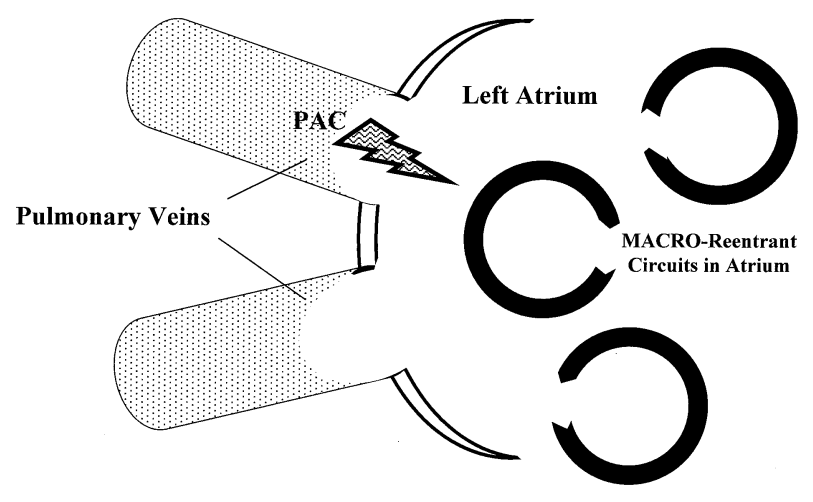

Figure 1. Induction of atrial fibrillation by a premature atrial beat originating in the orifice of one of the pulmonary veins. These triggers can be caused by microreentrant circuits that occur in the transitional zone of cells as the pulmonary vein endothelium transitions into the left atrial endocardium. However, it is most likely that these triggers are automatic rather than reentrant because recurrent atrial tachycardias are known to arise from automatic foci in other parts of the atria. ${ }^{9}$ PAC, Premature atrial beat.

Indeed, the clinical presentations are different specifically because the underlying electrophysiology is different. This correlation explains why their interventional treatment must also be different. Thus this classification system is accurate clinically, electrophysiologically, and therapeutically, and the terminology is simple, descriptive, accurate, and logical. Moreover, the terms continuous and intermittent are not only specific and uniformly understandable, but their meanings are direct opposites (ie, they are each other's antonyms).

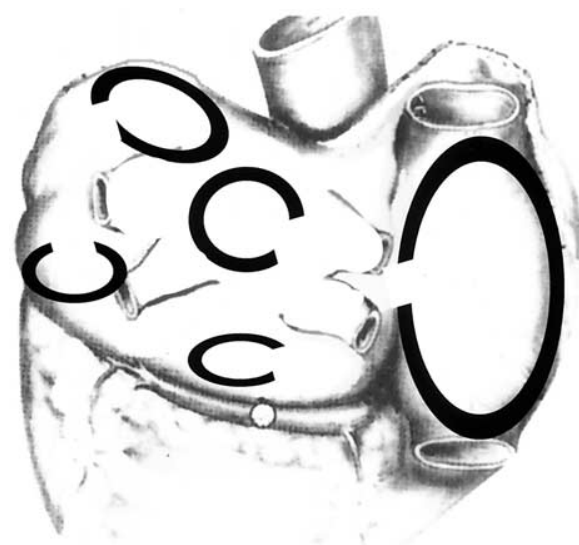

Figure 2. Once induced, all atrial fibrillation is characterized by the presence of multiple macroreentrant circuits in the atria. This electrophysiologic basis of atrial fibrillation is unrelated to (1) how or from where it was induced, (2) whether it is intermittent or continuous, and (3) its duration. 


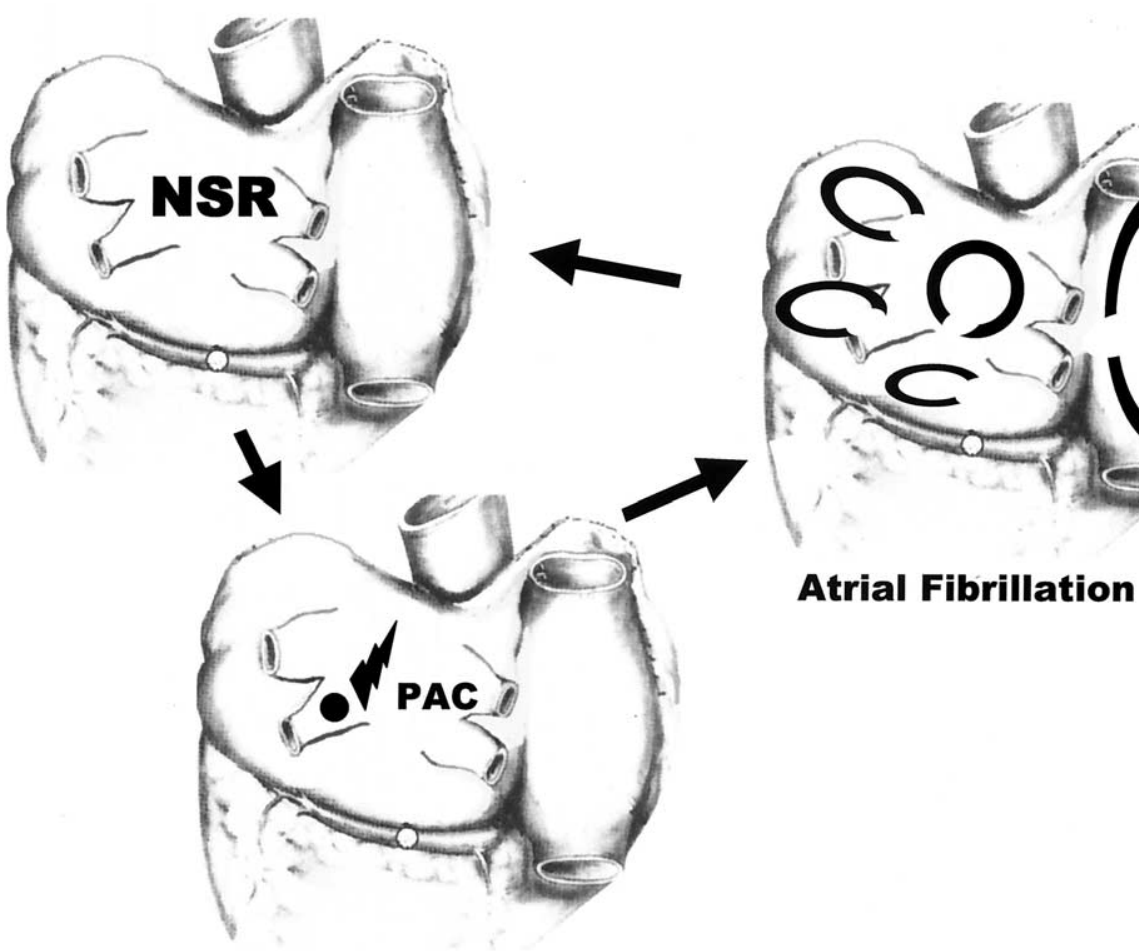

Figure 3. Intermittent atrial fibrillation (see text for further discussion). NSR, Normal sinus rhythm; PAC, premature atrial beat.

\section{Electrophysiologic Basis Intermittent Atrial Fibrillation}

It is intuitive that atrial fibrillation that is intermittent has to be induced repeatedly and thus requires a trigger to induce each individual episode of atrial fibrillation. In 1998, Hais-

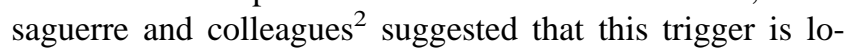
cated in the orifices of the pulmonary veins in approximately $90 \%$ of patients, although more recent studies have suggested that Haissaguerre et al's figure is much too high. ${ }^{3}$ Regardless of the true incidence of pulmonary vein triggers, the resultant premature atrial beat originating within the pulmonary vein discharges into the left atrium and induces the formation of multiple macroreentrant circuits involving both atria (Figure 1). ${ }^{4}$ The multiple macroreentrant circuits cause the atria to quiver, a state we know as atrial fibrillation (Figure 2)..$^{5}$

Once induced, the atrial fibrillation persists until the macroreentrant circuits terminate spontaneously or are terminated by drugs or interventional therapy. If the atrial fibrillation terminates spontaneously, the patient automatically reverts to normal sinus rhythm (Figure 3 ). Each subsequent episode of atrial fibrillation then requires another premature atrial beat to initiate the episode, with the trigger again being the pulmonary veins in the majority of cases.

At least $10 \%$ of patients with intermittent atrial fibrillation have a triggering mechanism that does not involve the pulmonary veins (Figure 4). ${ }^{2,3}$ It is well documented that atrial fibrillation can be induced intermittently by the Wolfe-Parkinson-White syndrome, atrioventricular node reentry, or deterioration of atrial flutter, ${ }^{5,6}$ but in most cases these nonpulmonary vein triggers, like their counterparts in the pulmonary veins, are simple automatic foci located elsewhere in the atria.

\section{Continuous Atrial Fibrillation}

If normal sinus rhythm cannot be reestablished either spontaneously or with drugs, the atrial fibrillation will no longer be episodic, and once induced, the atria will continue to fibrillate indefinitely. This is by definition continuous atrial fibrillation. Once atrial fibrillation becomes continuous, it does not need a trigger to reinduce it repeatedly because the atria are fibrillating all of the time. Thus continuous atrial fibrillation does not depend on the pulmonary veins for induction or perpetuation.

The reason that atrial fibrillation becomes continuous is because the macroreentrant circuits in the atrial myocardium have the ability to sustain themselves. Allessie ${ }^{7}$ has attributed this to atrial remodeling. This is electrical remodeling and might or might not be associated with anatomic remodeling (ie, with enlargement, hypertrophy, or stretching of the atria). The essence of this concept, as Allessie points out, is that "atrial fibrillation begets atrial fibrilla- 


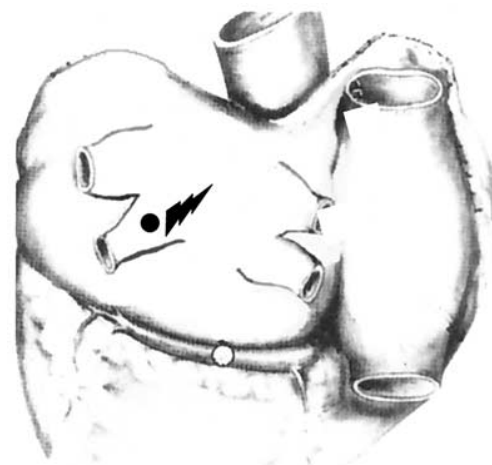

$90 \%$ from Pulmonary Veins

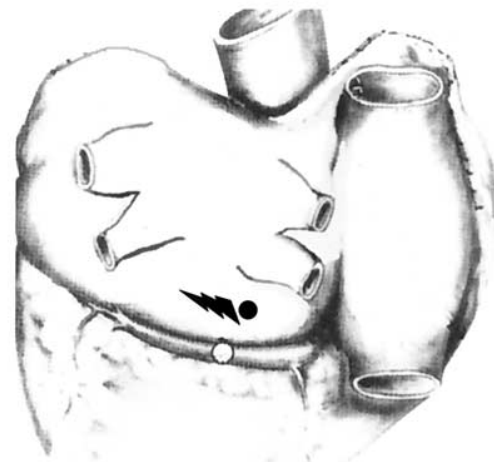

$10 \%$ Outside Pulmonary Veins

Figure 4. The trigger for the induction of intermittent atrial fibrillation is located in the pulmonary veins in $90 \%$ of patients and outside the pulmonary vein area in $10 \%$ of patients.

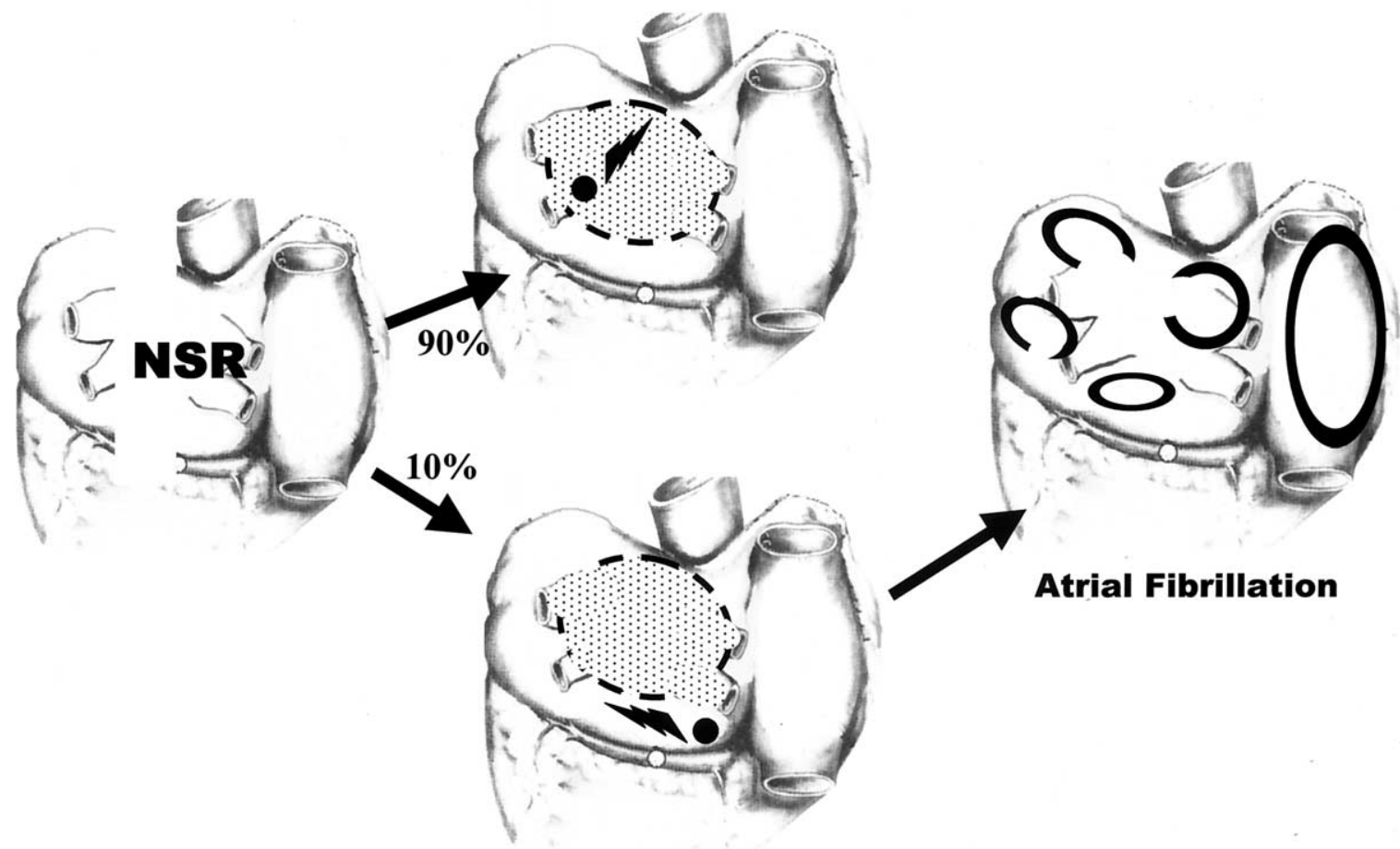

Figure 5. Pulmonary vein isolation for intermittent atrial fibrillation. Because the trigger resides within one or more of the pulmonary veins in $\mathbf{9 0} \%$ of patients with intermittent atrial fibrillation, simple pulmonary vein encirclement will cure $\mathbf{9 0} \%$ of these patients. However, $\mathbf{1 0} \%$ of patients with intermittent atrial fibrillation will not be cured with simple pulmonary vein isolation. NSR, Normal sinus rhythm.

tion." In other words, the more a patient experiences atrial fibrillation, the more that patient is likely to experience it again.

\section{Interventional Therapy}

Simple pulmonary vein encirclement confines the trigger mechanism for intermittent atrial fibrillation to the pulmo- nary veins, and therefore if performed adequately, it will cure the majority of patients with intermittent atrial fibrillation (Figure 5). Unfortunately, it is doomed to fail as an isolation procedure in a significant number of patients with intermittent atrial fibrillation because the trigger mechanism is not in the pulmonary veins. In addition, because continuous atrial fibrillation is not dependent on the pulmonary 


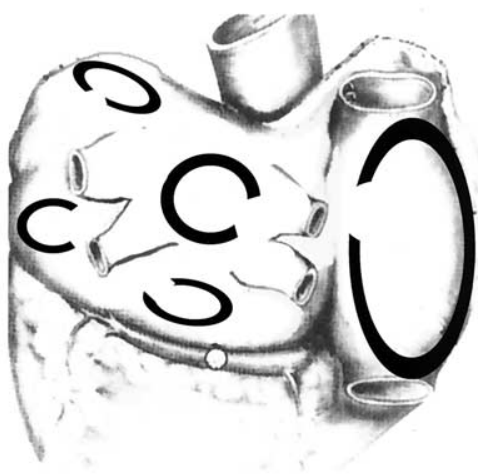

Continuous Atrial Fibrillation

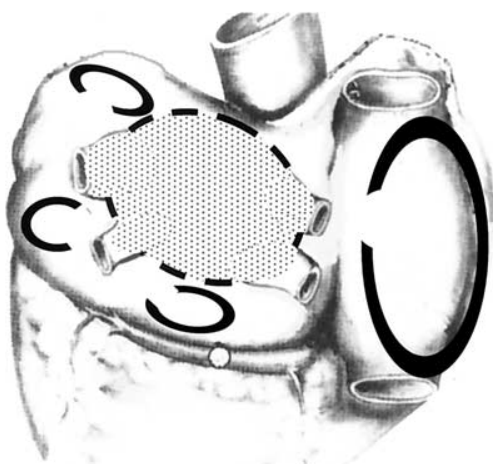

Pulmonary Vein Isolation

Figure 6. Failure of pulmonary vein isolation in patients with continuous atrial fibrillation. Because the maintenance of continuous atrial fibrillation is not dependent on induction triggers in the pulmonary veins, simple pulmonary vein isolation in these patients is not scientifically sound as an interventional-surgical approach to treatment.

veins for its maintenance, simple pulmonary vein encirclement is not a logical treatment goal in patients with continuous atrial fibrillation (Figure 6).

Thus in some patients with intermittent atrial fibrillation and in all patients with continuous atrial fibrillation, simple pulmonary vein encirclement is inadequate, and the goal shifts from isolating the trigger to ablating the macroreentrant circuits that characterize atrial fibrillation. One way of accomplishing the latter would be to "breadloaf" the atria, but obviously that would destroy the function of the atria postoperatively. Another way of precluding the possibility of atrial macroreentry (and thus atrial fibrillation) would be to excise enough of the atrial mass to make it too small to sustain macroreentrant circuits, but again, its function postoperatively would be destroyed. The only feasible way to ablate the atrial macroreentry and at the same time leave the resultant sinus rhythm capable of activating the entire atrial myocardium to ensure postoperative atrial transport function is to perform either the maze procedure or some variant thereof. The maze procedure is a classic ablation procedure in that it abolishes the macroreentry responsible for atrial fibrillation. Its lesion patterns are complex only because of the requirement that it not destroy postoperative atrial transport function.

\section{Observations that Modify the Rules}

The above discussion can be simplified to the following rules:

1. The objective of interventional-surgical treatment in the majority of patients with intermittent atrial fibrillation is to isolate the inducing trigger or triggers to the pulmonary veins.

2. The objective of interventional-surgical treatment in patients with continuous atrial fibrillation is to ablate the macroreentrant circuits responsible for its maintenance.

As with all rules and classifications, there are occasional exceptions that can aggravate the level of confusion. For example, one clinical observation that is problematic for the simple classification and treatment regimen outlined above is that pulmonary vein isolation alone does occasionally cure continuous atrial fibrillation. The principles described above would suggest that to be impossible.

Because of the confusion involving the meaning of the term chronic, as discussed above, it is impossible to know exactly how many patients with continuous atrial fibrillation have been cured by means of pulmonary vein encirclement alone, although it is clear that the total number is relatively small. Nevertheless, in view of the known electrophysiology described above, a legitimate question to raise is how simple pulmonary vein encirclement can ever cure a patient with continuous atrial fibrillation. There would seem to be 2 plausible explanations.

First, if an unusually large area of the atrial cuff is included within the pulmonary vein encircling incision, so much of the left atrium might be isolated that all of the functional macroreentrant circuits in the left atrium of that particular patient might be inadvertently interrupted or isolated. This physiology is equivalent to that occurring with excision of a large portion of the left atrium. The problem with this seemingly fortuitous scenario is the risk that so much of the left atrium will be isolated that the remaining left atrial function might be essentially destroyed.

Second, it has recently been suggested that in some patients the triggers in the pulmonary veins might fire incessantly, thereby continuously triggering the atrial fibrillation so that every time the arrhythmia tries to break, it is immediately reinduced. ${ }^{8,9}$ Therefore despite having the 
physiology of intermittent atrial fibrillation, this scenario would produce the clinical picture of continuous atrial fibrillation, but it would be curable by means of simple pulmonary vein isolation. Because continuous rapid firing of an automatic ectopic focus has been documented to occur in other parts of the left atrium, it is reasonable to suspect that it might occur in the region of the pulmonary vein orifices as well. If such an incessant trigger constantly precluded the patient from converting to sinus rhythm for more than 1 or 2 beats and if it were present within or near the orifices of the pulmonary veins, then pulmonary vein encirclement would cure this particular case of continuous atrial fibrillation.

Although the first scenario likely occurs clinically, there is little reason to believe that the second scenario occurs with any significant frequency. In 35 years of experience with operations for all types of cardiac arrhythmias, the author has encountered only one patient with long-standing, unrelenting, continuous rapid firing of an atrial ectopic focus, that occurring in the left atrial appendage of a 14 year-old boy. ${ }^{4}$ This incessant firing of the automatic focus did not cause atrial fibrillation in that patient but rather produced a rapid (200 beats/min) regular atrial tachycardia that remained unabated for 4 years until it was surgically terminated by means of cryoablating the trigger at the tip of the left atrial appendage. The rarity, indeed the virtual absence clinically, of this type of continuous trigger mechanism elsewhere in either atrium makes it extremely unlikely that it would occur in or around the pulmonary veins. In addition, unless the substrate already exists for the maintenance of macroreentry within the atria, there is no reason to believe that such pulmonary vein tachycardia would cause atrial fibrillation rather than a rapid regular atrial tachycardia. Indeed, the reports of this pulmonary vein tachycardia describe bursts of rapid tachycardia within the pulmonary veins rather than the incessant, unrelenting trigger that would be necessary to make physiologic intermittent atrial fibrillation present clinically as continuous atrial fibrillation. Thus even though simple encirclement of the pulmonary veins in patients with continuous atrial fibrillation might occasionally inadvertently cure the problem, it is not a scientifically sound surgical-interventional approach in such patients.

\section{The Rationale for Future Interventional Therapy}

Although predicting the future is always perilous, enough facts are available to know that the clinical spectrum of atrial fibrillation is destined to change in the coming years. Of the approximately 3000 patients with atrial fibrillation whom the author personally interviewed over the past 20 years, approximately one half had intermittent atrial fibrillation, and one half had continuous atrial fibrillation. Those patients fell into the following historical categories.
Patients presenting with intermittent atrial fibrillation (approximately $50 \%$ of the total) were defined as patients who had experienced only intermittent atrial fibrillation

Patients presenting with continuous atrial fibrillation (approximately $50 \%$ of the total) were defined as patients who had intermittent atrial fibrillation initially that eventually deteriorated into continuous atrial fibrillation and patients who converted from normal sinus rhythm directly into continuous atrial fibrillation without a transition period of intermittent atrial fibrillation.

Among the roughly $50 \%$ of patients who presented in continuous atrial fibrillation, virtually all had experienced intermittent atrial fibrillation at some earlier point in their lives. As mentioned above, this evolution from intermittent to continuous atrial fibrillation occurs because of the phenomenon of atrial remodeling, in which the more a patient experiences atrial fibrillation, the more that patient is likely to experience it again.

In view of this observation, it seems apparent that as surgeons and interventional cardiologists become more aggressive and successful in treating intermittent atrial fibrillation, progressively fewer patients will ultimately be allowed to have continuous atrial fibrillation. Thus it follows that in the future, most patients who present for atrial fibrillation treatment will have the intermittent type, the majority of which is amenable to simple pulmonary vein isolation procedures. Nevertheless, it would seem that the ultimate goal of interventional-surgical therapy should be to cure all patients with atrial fibrillation, including those with enlarged atria, thickened atrial walls, or both and those with associated cardiac disease. The attainment of that goal requires a better understanding of the problem, more accurate reporting of results, and a diversified armamentarium of procedures, all of which must be based on sound scientific and surgical principles.

\section{References}

1. Fuster V, Ryden LE, Asinger RW, Cannom DS, Crijns HJ, Frye RL, et al. ACC/AHA/ESC guidelines for the management of patients With atrial fibrillation: executive summary. A report of the American College of Cardiology/American Heart Association Task Force on Practice Guidelines and the European Society of Cardiology Committee for Practice Guidelines and Policy Conferences (Committee to Develop Guidelines for the Management of Patients With Atrial Fibrillation) developed in collaboration with the North American Society of Pacing and Electrophysiology. Circulation. 2001;104(17):2118-50.

2. Haissaguerre M, Jais P, Shah DC, Takahashi A, Hocini M, Quiniou G, et al. Spontaneous initiation of atrial fibrillation by ectopic beats originating in the pulmonary veins. N Engl J Med. 1998;339(10):659-66.

3. Schmitt C, Ndrepepa G, Weber S, Schmieder S, Weyerbrock S, Schneider M, et al. Biatrial multisite mapping of atrial premature complexes triggering onset of atrial fibrillation. Am J Cardiol. 2002;89(12):1381-7.

4. Gallagher JJ, Cox JL, German LD, Kasell JH. Nonpharmacologic treatment of supraventricular tachycardia. In: Josephson ME, Wellens HJJ, editors. Tachycardias: mechanisms, diagnosis, treatment. 1st ed. Philadelphia: Lea \& Febiger; 1984. p. 271-85. 
5. Cox JL, Canavan TE, Schuessler RB, Cain ME, Lindsay BD, Stone C, et al. The surgical treatment of atrial fibrillation: II. Intraoperative electrophysiologic mapping and description of the electrophysiologic basis of atrial flutter and atrial fibrillation. J Thorac Cardiovasc Surg. 1991;101:406-26.

6. Canavan TE, Schuessler RB, Boineau JP, Corr PB, Cain ME, Cox JL. Computerized global electrophysiological mapping of the atrium in patients with Wolff-Parkinson-White syndrome. Ann Thorac Surg. 1988;46:223-31.
7. Allessie MA. Atrial fibrillation-induced electrical remodeling in humans: what is the next step? Cardiovasc Res. 1999;44(1):10-2.

8. Jais P, Weerasooriya R, Shah DC, Hocini M, Macle L, Choi KJ, et al. Ablation therapy for atrial fibrillation (AF): past, present and future. Cardiovasc Res. 2002;54(2):337-46.

9. Oral H, Ozaydin M, Tada H, Chugh A, Scharf C, Hassan S, et al. Mechanistic significance of intermittent pulmonary vein tachycardia in patients with atrial fibrillation. J Cardiovasc Electrophysiol. 2002; 13(7):645-50

\section{JTCVS On-Line Manuscript Submission and Review}

\section{Please visit http://www.editorialmanager.com/jtcvs/}

Effective September 15, 2001, authors and reviewers may submit manuscripts and reviews electronically via Editorial Manager, our new Web-based system with full electronic submission, review, and status update capabilities.

As we move from paper to electronic submissions, the Editorial Office will make proxy submissions of all manuscripts accompanied by a diskette containing the electronic files of the text, tables, and figures. Editors, authors, and reviewers will receive automatic e-mails when significant events occur.

We strongly encourage all authors and reviewers to use Editorial Manager. Although we will continue to accommodate the submission of paper manuscripts for some months, our goal is to be completely electronic within 9 to 12 months.

All individuals currently in our database for whom we have e-mail addresses will receive via e-mail a system-assigned username and password that can be used to log in to the system without prior registration. All those not receiving the e-mail must register the first time they use the system.

As with any broad systemic change, the conversion to the new system will take some time to complete. We ask your patience as we replace our in-office database with the new system. We also encourage you to take advantage of the speed and efficiency that the new system will

provide for us all: editor, author, reviewer, and publisher. 